\title{
An Analysis of the Optical System of a Length Measurement Comparator
}

\author{
Rimantas LAZDINAS*, Mindaugas JUREVICIUS**, Vladas VEKTERIS***, \\ Vytautas TURLA****, Eugenijus JURKONIS****** \\ *Vilnius Gediminas Technical University, Basanaviciaus 28, LT-03224 Vilnius, Lithuania, E-mail: r.lazdinas@gmail.com \\ **Vilnius Gediminas Technical University, Basanaviciaus 28, LT-03224 Vilnius, Lithuania, \\ E-mail: mindaugas.jurevicius@vgtu.lt \\ ***Vilnius Gediminas Technical University, Basanaviciaus 28, LT-03224 Vilnius, Lithuania, \\ E-mail:vladas.vekteris@vgtu.lt \\ ****Vilnius Gediminas Technical University, Basanaviciaus 28, LT-03224 Vilnius, Lithuania, \\ E-mail: vytautas.turla@vgtu.lt \\ *****Vilnius Gediminas Technical University, Basanaviciaus 28, LT-03224 Vilnius, Lithuania, \\ E-mail: eugenijus.jurkonis@vgtu.lt
}

cross'ref http://dx.doi.org/10.5755/j01.mech.26.6.24554

\section{Introduction}

The increasingly growing level of industry robotization causes a need in high accuracy bar length measures and the latter, in its turn, stimulates an acceleration of the processes of manufacturing such measures. However, it is impossible to implement in absence of high efficiency calibration of precise scales. Upon striving to ensure the required efficiency, new measurement system and methods that would enable to achieve a high accuracy of dynamic calibration upon non-ideal metrological conditions are being developed. The accuracy of detecting the bar position upon using a length or angle measurement comparator $[1,2]$ is considerably affected by the optical system of the comparator. The structure of an interferometer integrated with the mechanical structure presents one of the principal parts of the measuring system that enabled reducing the indeterminacy. Upon striving to avoid mechanical and thermal interference, the principal components of the interferometer were produced of materials with low thermal expansion coefficient. A ray from the source of radiation (a neodymium garnet laser stabilised with iodine) is transferred by the interferometer using single-mode optical fibre cables [3].

The interferometer enables to compensate elongations and deflections of the granite base. If such deformations appear during a measurement, they can cause Abbe errors while detecting the position of the line under calibration. The said deflections as well as the tilt of the body of the optical system of the interferometer are compensated by additional Michelson interferometer. When a contour for the carriage angular oscillation control was introduced, the said oscillations decreased to $0.15 \mu \mathrm{rad}$, thus enabling to reduce Abbe errors to negligibly small values $[4,5]$.

The review of the comparators of various structures and the algorithms for bar position detecting shows a broad variety of designs and structures of comparators. Comparators from 12 countries were classified according to groups of the principal units [6].

For transfer of a motion, the guides and the electric motor are used. Most frequently, a granite base with air bearings are used; slideway bearings go second and Teflon sliding bearings go third.
The bar detection hardware includes: a CCD (charge-coupled device) microscope and a split-field photoelectric scanning microscope. For bar detection, truncation of the profile on a certain level with linear approximation, gradient operators and other means are applied [7].

For bar detection (edge identification) in length calibration systems, a photoelectric microscope with CCD (charge-coupled device) or CMOS (complementary metal oxide semiconductor) sensors is used. The type of the used microscope predetermines the signal processing algorithm as well as the accuracy and the rate of calibration.

The efficiency of the detection optical system depends on the illumination method, the image sensor, optical transfer function, the image defocusing, and the image data processing algorithm [8].

The illumination of the object under measurement is almost the most critical and subtle aspect in microscopy upon striving for highlighting the properties of the specimens. The illumination of the system under analysis shall be optimized by establishing the interdependence between the illumination and the image parameters upon striving to ensure the maximum spatial resolution, sensibility and bar detection accuracy. In technical imaginary systems, three principal methods of objects' illumination are applied:

- episcopic illumination (reflected light),

- diascopic illumination (transmitted light),

- structurised illumination.

Frequently reflected light and transmitted light are applied simultaneously upon striving to highlight specific properties of the specimen. To increase the image contrasting, the illuminators are equipped with filters, prisms, polarisers, analysers and other optical elements [9].

The task for this work was formulated as follows: to identify both edges of the digital signal. If the positions of the signal edges are known, the position of the signal centre may be calculated.

If the linear approximation method is applied for signal edges approximation on a certain level (most frequently, from $30 \%$ to $70 \%$ of the maximum amplitude of the signal), the signal edge may be approximated by a straight line. The parameters of the straight line (the directional factor $\beta$ and the leaning $\alpha$ ) are calculated upon applying the least squares method. In an ideal case, the directional 
factors of straight lines used for approximating the edges of profiles of repeating graduation (calibration) lines should be the same. In course of analysing the signal obtained from the register, it was found that that the directional factors calculated for each edge of the signal differ inconsiderably. So, in the further calculations, the values of the directional factor $\beta$ are considered the same for all edges of the signal approximated by straight lines [4].

\section{The object under investigation}

Modern bar length measures are produced of various shapes of the cross-section and various lengths - from several micrometres to several metres. The materials usable for their production include steel, invar, glass, glass ceramic as well as the latest materials, such as glass ceramic Zerodur. The mentioned materials are distinguished for very low thermal expansion coefficient. In addition, the measure graduation density may vary from $0.5 \mu \mathrm{m}$ (on scales of very high accuracy) to several millimetres and more. On calibration of bar measures by length comparators (Fig. 1), the distances are measured between the bar centres: however, in some cases, distances between edges of the line profile are measured. Digital measuring microscopes enable to ensure precise positioning of bar length measure calibration systems and to assess the quality of the bars and the accuracy of their disposition.

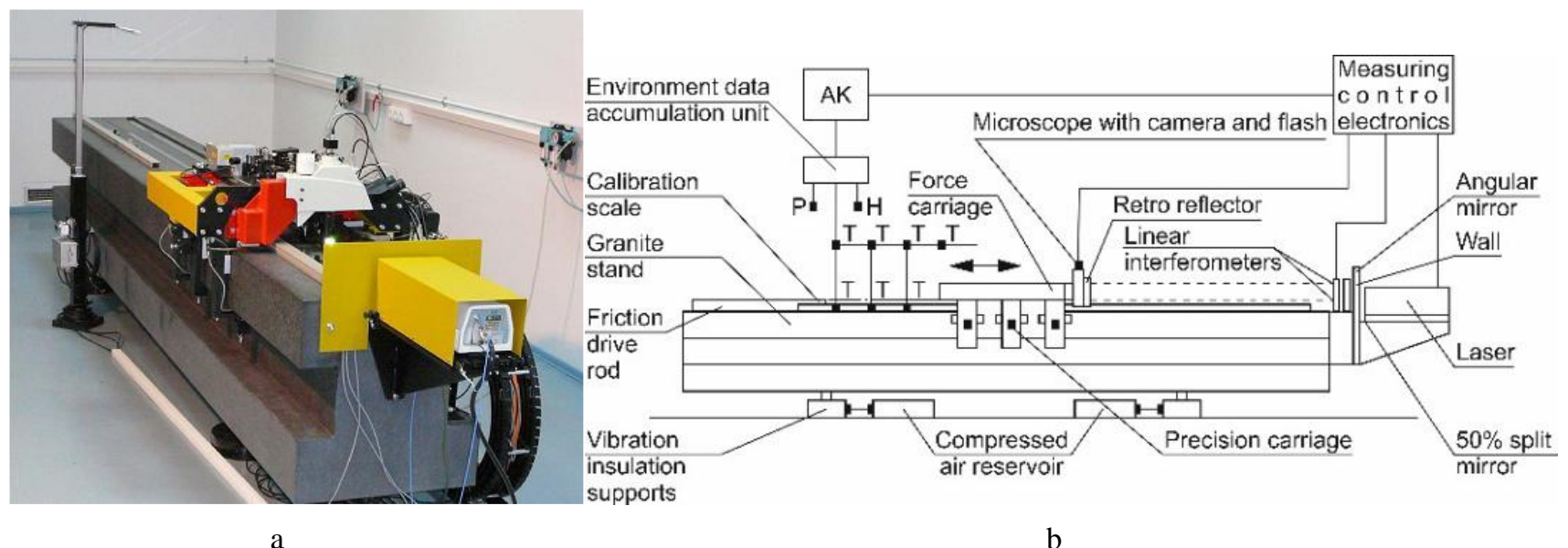

Fig. 1 Length scale comparator a) and its scheme

An additional optical interferometric system (installed on the granite guide close to the laser) equipped with error calculation software is involved in compensation of Abbe errors on a vertical plane. Abbe errors on a horizontal plane are inconsiderable. Along the laser ray, in ten fixed positions, temperature $\&$ pressure of the raster scale and air are measured. The measured values of temperature and pressure are collected by the data storage unit and sent to the principal computer. In addition, the thermal extension of the scale is compensated in real time.

\section{An analysis of the optical system}

The most important parameter of a microscope is its resolution, i.e. its ability to recognize the smallest parts of an object. An optical resolution is defined as a minimum distance between two point sources that allows recognizing them as two objects on image plane. No unique method for establishing the said resolution exists, because modern optical systems consist of abundant lenses with very different Image Point Spread Functions (hereinafter, the Lithuanian abbreviation VTIF is used for the said functions). Because the concept of resolution is bound with point sources, a resolution can be defined as VTIF recognisability. When VTIF spreads more than the resolution of the optical sensor allows, the observed objects become non-recognisable. Most frequency, a resolution is characterized by two parameters: Abbe and Rayleigh distances:

$$
r_{\text {Airy }}=1.22 r_{o}=0.61\left(\frac{\lambda}{N A}\right) .
$$

Abbe distance $r_{A b b e}$ is the diameter of VTIF central peak half of amplitude. The criterion of resolution formulated by Rayleigh means that two signals from point sources (situated on the plane of the focus) will be recognised by VTIF, if the distance between their peaks exceeds $r_{\text {Airy }}$.

For image shooting system, special Nikon objectives (for measurements) with 20x and 50x magnification whose digital apertures NA equal to 0.42 and 0.75 , respectively [10], are used. The shortest wavelength in the illumination system is $0.38 \mu \mathrm{m}$, so the result of calculations according to the formula (1) is following: $r_{A b b e}=0.45 \mu \mathrm{m}$ and $r_{\text {Airy }}=0.55 \mu \mathrm{m}$ for 20x magnification; $r_{A b b e}=0.25 \mu \mathrm{m}$ and $r_{\text {Airy }}=0.31 \mu \mathrm{m}$ for $50 \mathrm{x}$ magnification. This means that if a raster image illuminated by light with the wavelength of $0.38 \mu \mathrm{m}$ is observed by a microscope, when the frequency of raster structure is $2.2 \mathrm{bar}$ period/ $1 \mu \mathrm{m}$, the bars are still recognisable.

If the said parameters are known, the size of pixel for the sensor of the camera can be chosen. The required image discretization frequency should be no less than the optical resolution at the middle wavelength. For this reason, a digital video camera with $6.4 \mu \mathrm{m}$ pixel of the sensor was chosen. At 20x magnification, the image of the object is discretized in $0.32 \mu \mathrm{m}$ intervals, or 1.5 bar raster structure period/ $1 \mu \mathrm{m}$. So, the Rayleigh criterion is satisfied.

One more requirement is obligatory for the optical system: the signal discretization frequency should be at least twice as much as the maximum frequency of the signal. Because the minimum width of a precise scale's bar is $2 \mu \mathrm{m}$, it may be stated that this requirement is satisfied as well.

The above-mentioned calculations are approximate, because a complete evaluation requires supplemental 
data, such as the object's surface reflection, permeability, optical density characteristics and the intensity of illumination flow.

An objective is a complicated optical system that's VTIF is not known. In addition, VTIF is hard-to-measure. As it was mentioned above, the optical transfer function (OTF) is the Fourier transform of VTIF, so the inverse Fourier transform of VTIF is usable for VTIF establishing. Manufacturers of objectives usually provide only modulation transfer function (MTF) that is an amplitude function of OTF. In microscopy, the changes of the phase of waves after their passing through the optical system usually are not analysed. Therefore, only the amplitude function of OTF (in other words, MTF) is analysed. For its establishing, software of another author was used [11].

In addition, the objective blurs the image. Modern objectives are formed of many (over ten) different spherical lenses with various deflections of their shapes, so a mathematical description of the signal distortion by such a complicated optical system is almost impossible. So, manufacturers of objectives usually provide the modulation transfer function (MTF) of lenses of the objective. It is an amplitude transfer function of the objective's optical system, because the sensors do not fix phase changes. In a general case, in the equations that describe the image burring, namely th Fourier transform of the transfer function (in other words, VTIF) is used [3]:

$$
B=V T I F \otimes I+n,
$$

here: $B$ is the obtained image; VTIF is a function of spreading of the image's point; $\otimes$ is the convolution operator; $I$ is the ideal contrast image; $n$ is the additive white Gaussian noise. tions $[6]$

VTIF may be analysed as a product of several func-

$$
V T I F=V T I F_{\text {opt }} * V T I F_{m o t} * V T I F_{d e t} * V T I F_{e l},
$$

here: $V T I F_{\text {opt }}$ is VTIF of the optical system; VTIF mot is VTIF caused by motion; $V T I F_{d e t}$ is VTIF of the detector; VTIF $e l$ is quantisation and filtering VTIF.

Image blurring of the optical system is the largest, because it consists of abundant components (lens aberrations), including the component of image blurring caused by defocusing that is variable and the most difficult to detect. The component of image blurring caused by defocusing in the system under investigation is larger, as compared to the component related to image blurring caused by motion, and considerably larger than other components. So, even if the detector's and quantisation point spread functions are neglected, a reconstruction of image blurring caused by motion is very complicated.

According to the recommendations provided in references [3, 12, 13], a model of image formation on its blurring by motion was developed. In the beginning, we suppose that the velocity of motion $v$ is a real positive value. If the camera does not rotate on its motion, we find that any point of the image shown by the camera moves with the same velocity. The velocity is calculated using the pixel coordinates $x, y$ and time $t$. If the velocity is constant, it may be expressed by a two-dimensional motion vector $s_{x, y}=$ $\left[s_{x}, s_{y}\right]$. The image blurring by motion is a convolution of the blur kernel and the primary image [14]:

$$
B=\varphi_{s_{x, y}} \otimes I+n,
$$

here: $\varphi_{S x, y}$ is the point spread function of motion-blurred image (in other words, the blur kernel).

The values of the mentioned blur kernel depend on the motion of the camera. Mathematically, a convolution is a product of frequentative functions:

$$
\hat{B}\left(\omega_{x, y}\right)=\hat{\varphi}_{s_{x, y}}\left(\omega_{x, y}\right) \hat{I}\left(\omega_{x, y}\right)+\hat{n}\left(\omega_{x, y}\right),
$$

here: $\omega_{x, y}=\left[\omega_{x} \omega_{y}\right]$ is $2 \mathrm{D}$ frequency response and the sign ^ is the Fourier transform. It may be seen from this formula that when the component $\hat{\phi}_{s_{x, y}}\left(\omega_{x, y}\right)$ is small, a poor sig$\mathrm{nal} /$ noise ratio is obtained.

According to the conclusions provided in the reference [8], the quality of reconstruction of a motion-blurred image generally depends on the power spectrum $\left\|\hat{\phi}_{s_{x, y}}\left(\omega_{x, y}\right)\right\|^{2}$ of the discolouration kernel upon assessing the allowable reconstruction error or, in other words, the quality of reconstruction is bound with the total spectrum:

$$
\left\|\tilde{\phi}_{s_{x, y}}\left(\omega_{x, y}\right)\right\|^{2}=\sum\left\|\hat{\phi}_{s_{x, y}}\left(\omega_{x, y}\right)\right\|^{2} .
$$

The task of the reconstruction is establishing all the motions that increase the sum of spectrum power $\left\|\tilde{\phi}_{s_{x, y}}\left(\omega_{x, y}\right)\right\|^{2}$ for each frequency component $\left(\omega_{x, y}\right)$ and each motion vector $\left\|s_{x, y}\right\|$ smaller than the size of the picture.

Establishing the bar edge measurement indeterminacy is a more complicated task, as compared to measurement of the edge. It is supposed that the distribution of random errors of bar edge measurement is normal. Upon such a condition, the relative error of roughness measurement indeterminacy after $n$ bar edge measurements, when the roughness is expressed as standard deviation of the bar edge position, may be calculated according to the following formula:

$$
\frac{\Delta \sigma_{L E R}}{\sigma_{L E R}} \approx \frac{1}{\sqrt[2]{2 n}} .
$$

Bar contour roughness is one of the parameters describing the scale's bar. Roughness is defined as a deviation of values of the bar contour in respect of the bar edge.

The contour is a border between the light part and the dark part of the image. On the contour, vertical and horizontal surfaces contend. The contour shows striking changes of the image intensity; in our case, they are light reflecting bars and light transmitting gaps between them. In the realty, light is reflected is from gaps between surfaces and sweeps on the surface of the object under measurement as well, so additional reflections may cause distortions of the contour line.

The task of the contour detection by sub-pixel resolution is complicated. Abundant methods are applied for 
bar contour detection; however, it is difficult to single the most effective among them. Each method is distinguished for certain peculiarities that the quality of the contour and the accuracy of its detection depend on.

To ensure no less than 5\% accuracy of bar edge roughness measurement, at least 200 values of the bar contour should be measured. It is found from a single photo, because a sensor of the digital camera fixes a photo by $1024 \times 1280$ pixel matrix. It was calculated that when the total size of the sensor's matrix $(1024 \times 1280)$ is used and the relevant values are inserted in the formula (7), the relative error of the roughness measurement indeterminacy would not exceed $2.2 \%$.

Because the bar contour measurement is accomplished by a digital camera upon very short (up to $20 \mu \mathrm{s}$ ) image fixation and strong illumination, the accuracy of bar edge measurement equals to the sum of standard deviations of the bar contour positions and the camera's sensor noise.

Thus, the deviation of roughness of the edge under measurement shall be calculated as follows:

$$
\sigma_{\text {measured }}^{2}=\sigma_{L E R}^{2}+\sigma_{\text {camera noise }}^{2} .
$$

When a width of the bar is measured, the camera's sensor noise is averaged in course of processing by the measurement algorithm. Its values are positive only. It is stated that the impact of the averaged noise on calculations of the both edges of the bar is the same, so it is insignificant.

Noise of the camera depends on the conditions of operation of charge-coupled device (CCD) or complementary metal oxide semiconductor (CMOS) element. Usually the noise sources shown in Fig. 2 are being analysed. In the image element, free electrons randomly appear and the so called "darkness" current $i_{d c}$ begins flowing. It is the predominating noise of the sensor. In addition to it, a temporary noise (of a fixed pattern) and a noise of the reading circuit exist.

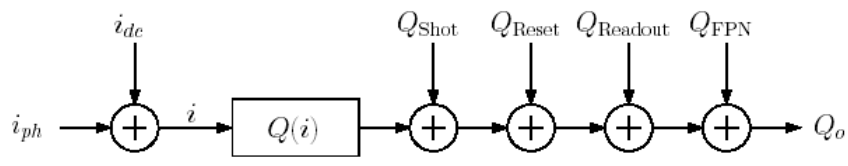

Fig. 2 Transfer model of photocurrent $i_{p h}$ to charge $Q_{o}$

Here: $Q_{i}$ is the sensor's current transformation function; $\mathrm{Q}_{\text {shot }}$ is the shot noise charge that depends on the integration time and that's average power is $i\left(i_{p h}+i_{d c}\right) t_{i n t} / q$; $Q_{\text {Reset }}$ is the fixed pattern deletion noise; $Q_{\text {Readout }}$ is the noise of the reading circuit; $Q_{F P N}$ is the additive noise of a fixed pattern that equals to:

$$
Q(i)=\left\{\begin{array}{cc}
\frac{1}{q}\left(i t_{\text {int }}\right), & 0<i<\frac{q Q_{\text {sat }}}{t_{\text {int }}}, \\
Q_{\text {sat }} & i \geq \frac{q Q_{\text {sat }}}{t_{\text {int }}}
\end{array},\right.
$$

here: $Q_{\text {sat }}$ is the sensor's saturation noise.

The longer image exposition time $t_{\text {int }}$, the higher evenness of noise distribution in CCD matrix will be. According to the image with a long exposition time, it is possible to form a map of CCD sensor's noise (because it acquires a character of a systematic error) and to compensate it partially. However, in the camera under investigation where the image exposition time was only some more than ten microseconds this noise should be considered a random error. So, this method of compensation is not applicable for elimination of the impact of noise on the bar edge roughness.

Another important factor affecting the accuracy of bar edge measurement is bound with frequency parameters of the bar edge, not with the amplitude ones. The edge is discretized into intervals of the pixel size divided by the microscope objective magnification. In such an interval, the values of the bar edge are optically averaged. In such a way, the indicator of bar edge roughness is improved.

For reading the image fixed in the camera's sensor by an intermediate buffer, libraries of special functions are used for transmission of the image. Packages of programming tools can read the said libraries and apply their functions. They are included in the bank of the key resources of the control programme.

\section{Results and discussion}

On measuring the bar width, a traditional algorithm with the signal "truncating" on the chosen level is most frequently applied. Prior to the calibration, the camera is rotated around the optical axis upon striving to obtain a bar image where the edge lines are almost parallel with a column or a line of photocells of the camera's sensor. The obtained two-dimensional image of slightly turned bars is suitable for processing according to this algorithm.

While using the algorithm with a limited level, it is necessary to obtain a two-dimensional function from the saved three-dimensional image; such a function should define the profile of the bar of the averaged intensity of the said three-dimensional image. If a vertical bar is explored, the saved values of the matrix in each column are summarized; then the result is divided by the number of lines and the averaged curve of intensity, otherwise referred to as the bar profile, is obtained. The photo of bars and the calculated profile are shown in Fig. 3. For this bar profile, a limited level should be established.

A limited level may be established as a constant level or on the base of the width of the range of the obtained bar profile values. For establishing a limited level, the criteria of the minimum and maximum values are often used. If the profile line is not even, the criteria of averaged maximum and averaged minimum values are applied. Frequently, the values of the peaks of the profile line are increasing or decreasing in respect of the axis X (they are referred to as puffs). In analysis of raster scales, only a part of the camera's sweep (not the total sweep) with four or five bars, i.e. about $240-360$ pixels, at raster step of $20 \mu \mathrm{m}$ is often used, as is shown in Fig. 3, a.

The peak values of the profile curve increase because the scale surface is not perpendicular to the optical axis of the microscope. Therefore, the values of the puff points are found and they are approximated by a straight. The said points are shown in Fig. 3, b, where the coordinates of the shot picture are shown in pixels on the $x$ axis of coordinates and the values of averaged columns of picture pixel intensity - on the $y$ axis of coordinates.

The profile curve shall be transformed in a way that ensures the intensity equalisation according to the approximating straight upon multiplying by the calculated elevation 
angle $\alpha$ and assessing the value by the spread coefficient. The scheme of profile curve transformation is provided in Fig. 4 below.

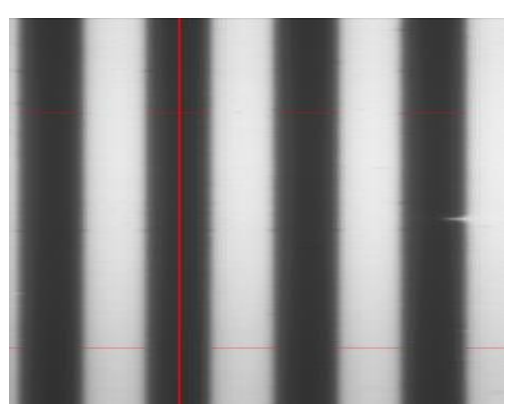

a

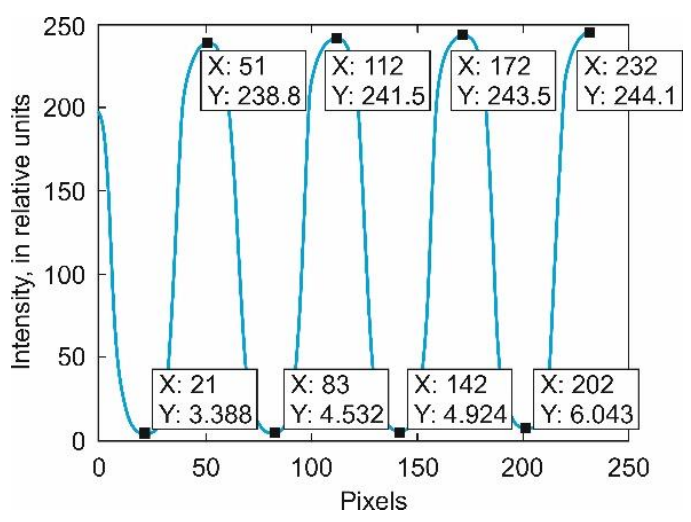

b

Fig. 3 Linear scale image of the detected line middle position (a) and the profile curve with peak values (b)

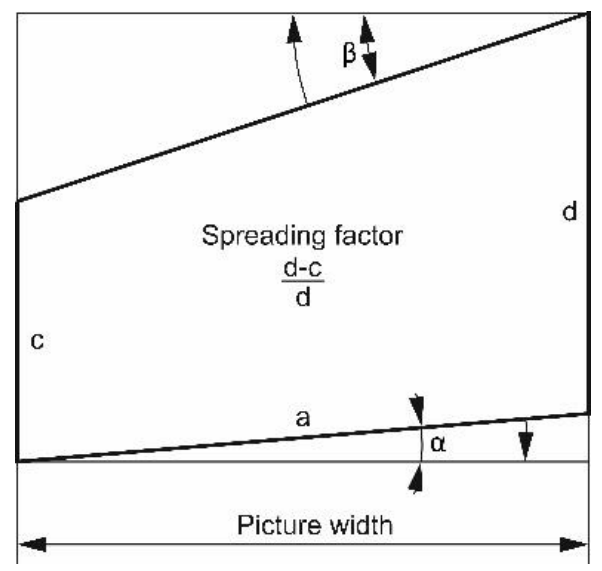

Fig. 4 Transformation scheme of image intensity profile

The peaks' approximating straights are used for calculation of the norming coefficient and the spread coefficient. The obtained image is multiplied by the said coefficients according to the following formula:

$$
\begin{aligned}
& A_{\text {pProle }}\left(i_{\text {pro }}\right)=\operatorname{ProIe}\left(i_{\text {pro }}\right)-b i_{\text {pro }}- \\
& -\frac{(d-c)}{d} \operatorname{ProIe}\left(i_{\text {pro }}\right) i_{\text {pro }},
\end{aligned}
$$

here: $A_{\text {pProle }}\left(i_{p r o}\right)$ is the $i$-th value of the equalized image intensity; ProIe $\left(i_{\text {pro }}\right)$ is the $i$-th value of the non-equalized image intensity; $b$ is the norming coefficient; $(d-c) / d$ is the spreading factor.
After equalization of the intensity profile curve (Fig. 4), the image shown in Fig. 5 is prepared for the bar edge detection:

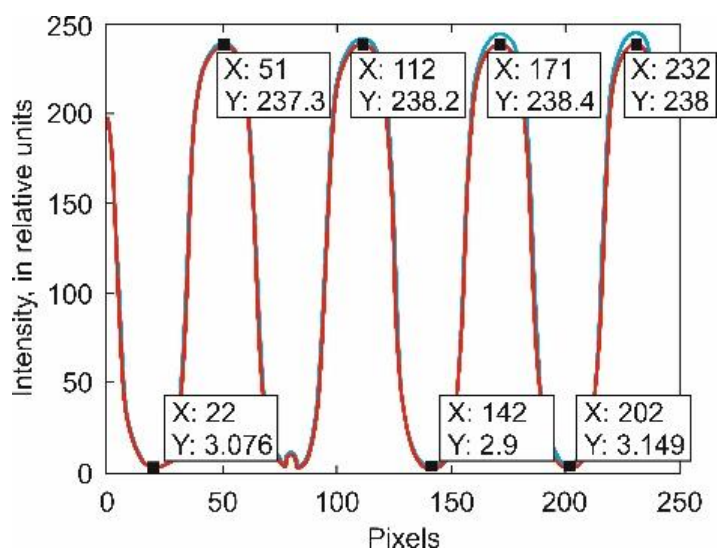

Fig. 5 Image intensity profile before transformation (blue) and after (red)

The developed bar edge detection method is, first of all, oriented to the scale calibration accuracy and high operating speed. In bar measurements, the curve of the bar profile is often used: one point of the contour (that is the average value of the contour) is found from the said curve, so hereinafter it is defined by the bar edge.

If the scale is calibrated upon applying the dynamic method, the quality of photos is worse, as compared to the static method. In the photos, a part of information is lost, as the object is out of the plane of the focus because of movement, short exposure interval, strong illumination and other factors. In the zone of the bar contour, the intensity is varying gradually. Therefore, shape of the bar profile curve is not obtained rectangular (as should be in the ideal case); it appears to be almost identical to a sinusoidal function. In addition to the above-listed factors, it may be caused by defects of the bar edge, deflections of the bar shape and a bar declination [15].

The positions of bars on raster scales may be analysed as a periodical sequence. A periodical sequence of rectangular (square-wave) impulses shall be mathematically described by the following formula:

$$
f(x)=\left\{\begin{array}{ccc}
-1, & \text { if }-\pi \leq x \leq 0 \\
1, & \text { if } \quad 0 \leq x \leq \pi
\end{array} .\right.
$$

In mathematical analysis, the following expression outspread by the Fourier series is more frequently applied:

$$
x_{\text {square }}(t)=\frac{4}{\pi} \sum_{k=1}^{\infty} \frac{\sin ((2 k-1) 2 \pi f t)}{(2-1)} .
$$

However, the shape of the real signals is almost identical to the shape of a sinusoidal function, so it may be outspread by the Fourier series as follows:

$$
\begin{aligned}
& f(x)=\frac{a_{o}}{2}+\sum_{n=1}^{\infty}\left[a_{n} \cos (x)+b_{n} \sin (n x)\right]= \\
& =2 \sum_{n=1}^{\infty} \frac{(-1)^{n+1}}{n} \sin (n x) .
\end{aligned}
$$


In the calculations, only several first members of the Fourier series are used. Upon applying the Fourier series, the positions of several bars from the raster scale can be assessed at once; this enables to reduce scattering of values of the scale under calibration and, in addition, averages the position of the bar of interest in respect of other bars. Therefore, such an analysis is suitable for raster scales only, when raster interaction is analysed.

After the equalisation according to the formula 10 and norming the values of the picture intensity profile, a bell-shaped curve was obtained. Its values vary between zero and one. In such a case, the comparing threshold will be on the level of 0.5 of the relative intensity, as shown in Fig. 6. Such an algorithm is applied in a majority of comparators under research. The bell-shaped edges are truncated on the level of 30-70\% and the values of the formed range are approximated by straights. The points of intersection of the said straights with the comparing threshold are considered an edge of a bar [8]. Sometimes only a part of bar profile slop is approximated and individual limit levels are established for each bar edge [5].

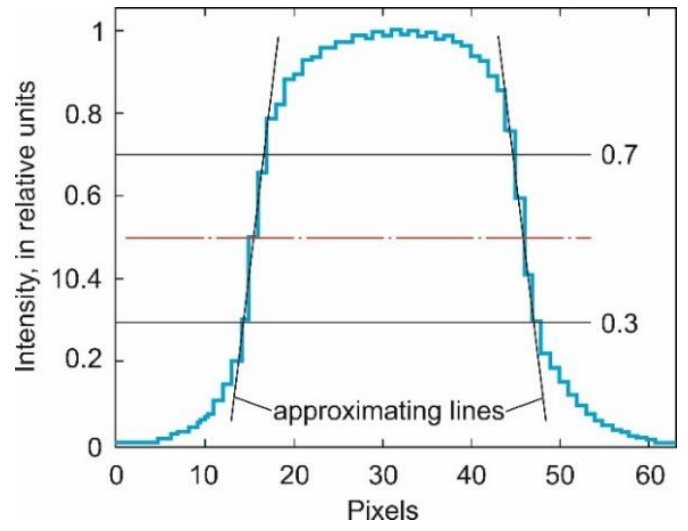

Fig. 6 Graph of line profile values and comparing threshold

The task related to bar edges is accomplished in several stages. The stage one is a "rough" establishment of bar edge from the profile curve. The pixel column that causes changes of the directions of the profile curve increasing or decreasing rates is found. For this purpose, two contour extraction methods (the gradient method and the second-order derivative method) are applied.

The gradient method is based on the first-order derivative of the function profile (Fig. 7) and establishing the local extremum. If the bar image is clear, the value of the derivative function peak is high. If the image is defocused and the signal/noise ratio is low, the peaks of the gradient are indistinct and several extremums may be found on a peak. This phenomenon reduces the accuracy of the edge identification. The second-order derivative method is even more sensible to local defects.

The aim of the first stage of the bar contour method under research is to identify the bar edges on the image with an accuracy to one pixel. For contour identification, digital filters based on the gradient or second-order derivative are used. Such digital filters are usable, if the values of the image under research are extracted by Wiener filter [16]. The image presented in Fig. 8, b is obtained after raster image processing by Wiener and Canny filters.

The said digital filters are based on a mathematical operation - convolution. A kernel of the convolution usually is a fragment of two-dimensional Gaussian function discretized by the size of the filter (see Fig. 9). Its values are whole numbers, the sides of the formed matrix are the same, and the values are symmetrical. The size of the filter is predetermined by the quality of the image under filtering, resolution of the contour to be identified and the parameters of the process rate. For the bar profile curve, convolution can be applied at once and such a mathematical operation would be very fast; however, in such a case, it would be impossible to assess the defects in the zone of the bar contour.

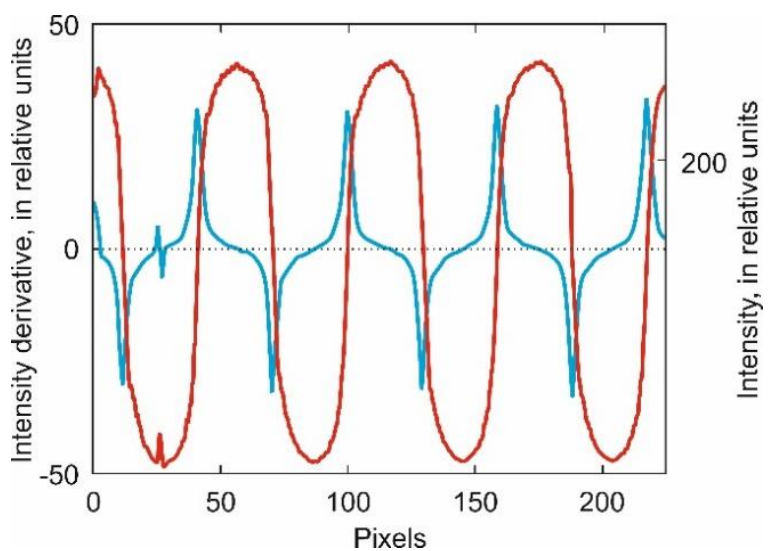

Fig. 7 Graph of line profile values and derivative of profile

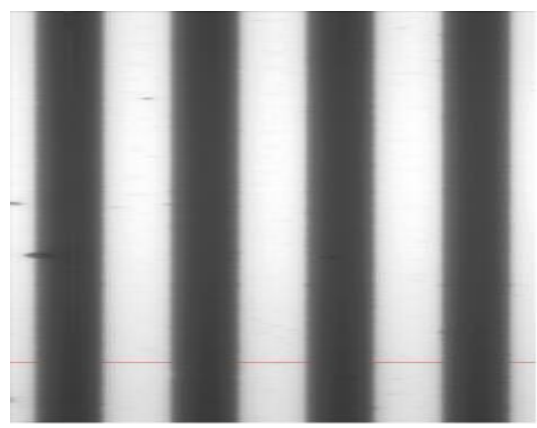

a

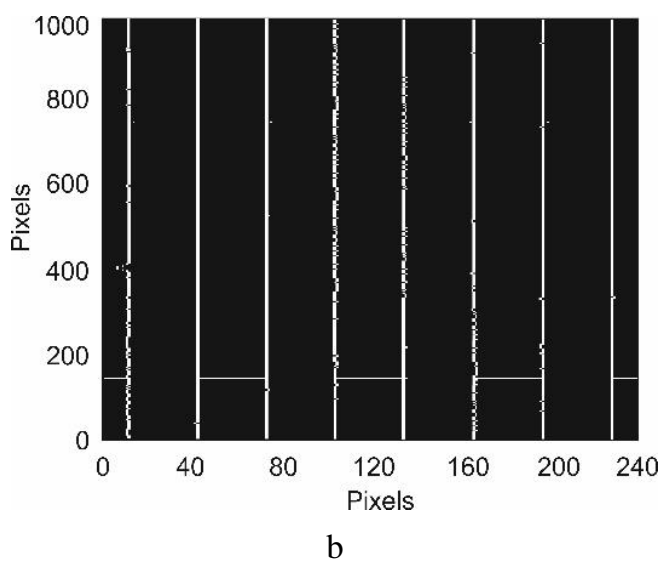

Fig. 8 Raster image (a); line edges extraction using Canny filtering (b)

When the bar contour is identified, it is possible to identify and "cut out" the fragments with defects [8]. Then the bar profile is formed again and a "fine" (precise) detection of the bar profile takes place. For a "fine" (precise) detection, images of subpixel resolution are required. There are two options of accomplishment of this provision: 1) to increase the number of pixels of the image, i.e. the resolution, 2) to apply alternative methods based on approximation of details of the pictures by functions $[17,18]$. 


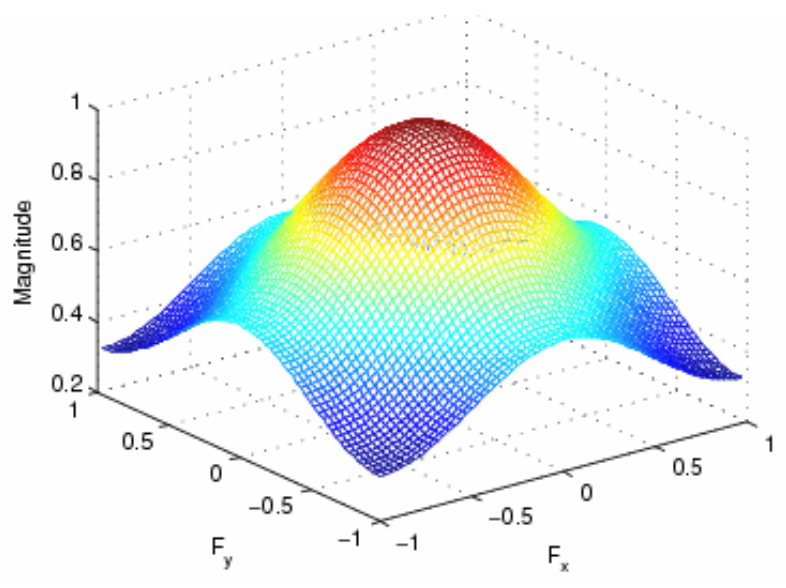

a

\begin{tabular}{c|c|c|c|c}
\hline 1 & 4 & 7 & 4 & 1 \\
\hline 4 & 16 & 26 & 16 & 4 \\
7 & 26 & 41 & 26 & 7 \\
\hline 4 & 16 & 26 & 16 & 4 \\
\hline 1 & 4 & 7 & 4 & 1
\end{tabular}

b

$\left(\begin{array}{ccccccccccc}0 & 0 & 0 & -1 & -1 & -2 & -1 & -1 & 0 & 0 & 0 \\ 0 & 0 & -2 & -4 & -8 & -9 & -8 & -4 & -2 & 0 & 0 \\ 0 & -2 & -7 & -15 & -22 & -23 & -22 & -15 & -7 & -2 & 0 \\ -1 & -4 & -15 & -24 & -14 & -1 & -14 & -24 & -15 & -4 & -1 \\ -1 & -8 & -22 & -14 & 52 & 103 & 52 & -14 & -22 & -8 & -1 \\ -2 & -9 & -23 & -1 & 103 & 178 & 103 & -1 & -23 & -9 & -2 \\ -1 & -8 & -22 & -14 & 52 & 103 & 52 & -14 & -22 & -8 & -1 \\ -1 & -4 & -15 & -24 & -14 & -1 & -14 & -24 & -15 & -4 & -1 \\ 0 & -2 & -7 & -15 & -22 & -23 & -22 & -15 & -7 & -2 & 0 \\ 0 & 0 & -2 & -4 & -8 & -9 & -8 & -4 & -2 & 0 & 0 \\ 0 & 0 & 0 & -1 & -1 & -2 & -1 & -1 & 0 & 0 & 0\end{array}\right)$

Fig. 9 3D Gaussian mesh (a); 5x5 convolution kernel (b); $11 \times 11$ convoliution kernel (c)

The method based on resolution increasing is often applied in microscopy, not in measurements. On increasing the resolution, an interpolation is applied and it causes an appearance of a new additional pixel between adjacent pixels. The amount of information from the picture increases (dependently on the method applied) from two to sixteen times.

In Fig. 10, the bar edge profile curve is presented. When its sides are truncated on the level of 30-70\% and the values of that range are approximated by straights, only 4 5 points of the curve remain involved in the calculations. In case of bar asymmetry or defect, the accuracy of the edge detection becomes worse.

In the work, a new algorithm for bar edge detection is proposed. After the "rough" detection of the value of the bar edge, we select six profile curve values before the said value and six values after it and approximate them by an algebraic $5^{\text {th }}$ degree polynomial. Then we establish the values of the polynomial on the comparing threshold. This method assesses the bar edge more precisely, in particular in presence of bar asymmetry or defect.

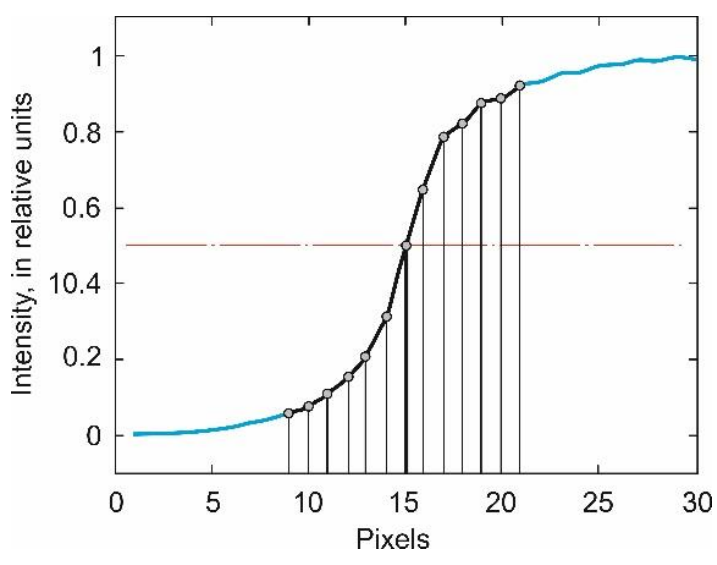

Fig. 10 Line left edge profile and profile values used for approximation

\section{Conclusions}

1. In course of an analysis of the comparator's structure, it was found that the components of indeterminacy of results of dynamic calibration of bar length measures are bound with distortions of the digital image caused by movement. The minimum error of bar length measures is obtained after optimization of the carriage rate, scale illumination, camera exposure time and its delay parameters.

2. After optimization of the calibration rate and the camera's parameters, the recommended calibration rate of 3 $\mathrm{mm} / \mathrm{s}$ for the friction gear and $6 \mathrm{~mm} / \mathrm{s}$ for a rope drawing mechanism were achieved when the camera exposure time was $17 \mu \mathrm{s}$. While calibrating the raster scale, 0.046-0.051 $\mu \mathrm{m}$ data scatter estimate was achieved. The maximum influence was caused by the carriage rate and the vibrations.

3. The obtained experimental results show that a detection of a sub-pixel displacement caused by movement is possible upon applying the proposed method; however, it will be only approximate whereas:

- $\quad$ it is necessary to know beforehand VTIF caused by defocusing and the image cannot be defocused more than for a half of Rayleigh scattering distance;

- noise impacts the detection of a displacement. If the noise energy exceeds $50 \%$ of the picture's energy, a detection of displacement by this method usually is unsuccessful;

- a displacement under 0.3 pixel is not detected by the proposed algorithm; with an increase of displacement, the accuracy of its assessment increases as well.

- for detection of displacement, 5 iterations are sufficient. A higher number of iterations does not improve the result.

4.The efficiency of the optical system related to illumination, image sensor, optical transfer function, image defocusing and image data processing algorithm had been established as well.

5.The results obtained in the work enable to improve the accuracy of dynamic calibration of bar length and angle measures.

\section{References}

1. Jurevicius, M.; Skeivalas, J.; Kilikevicius, A.; Turla, V. 2017. Vibrational analysis of length comparator, Measurement 103: 10-17. 
https://doi.org/10.1016/j.measurement.2017.02.010.

2. Kilikevicius, A.; Jurevicius, M.; Skeivalas, J.; Kilikeviciene, K.; Turla, V. 2016. Vibrational analysis of angle measurement comparator, Signal, Image and Video Processing 10(7): 1287-1294. https://doi.org/10.1007/s11760-016-0956-8.

3. Moghaddam, M. E.; Jamzad, J. 2007. Linear motion blur parameter estimation in noisy images using fuzzy sets and power spectrum, EURASIP Journal on Advances in Signal Processing 68985, 8 p. https:/doi.org/10.1155/2007/68985.

4. Flügge, J.; Köning, R.; Bosse, H. 2005. Characterising the performance of the PTB line scale interferometer measuring photoelectric encoders, Proc. SPIE, Recent Developments in Traceable Dimensional Measurements 5879: 08-1 - 08-9.

5. Gonzalez, H.; Galvan, C.; Muñoz, J. A. 2003. Image processing automatic interferometric calibration system for line scales, Proceedings of the SPIE 5190: 93-102.

6. Allebach, J. P. 2000. Image Scanning, Sampling, and Interpolation, Handbook of Image and Video Processing, 2nd edition, A. Bovik, editor, Academic Press, 629-643, ISBN 0-12-119792-1.

7. Bovik, A. C. 2005. Basic Tools for Image Fourier Analysis, in The Handbook of Image and Video Processing, Second Edition, New York, Academic Press, 57-72.

8. Družovec, M.; Acko, B.; Godina, A.; Welzer, T. 2009. Robust algorithm for determining line centre within a video positional measuring system, Optics and Lasers in Engineering 11(47): 1131-1138.

9. Goodman, J. W. 1996. Introduction to Fourier Optics, 2nd edition, McGraw-Hill book company, $491 \mathrm{p}$.

10. Nikon measuring microscopes. 2008. MM-400/800 series, Code No.2CEIDYH-4, 31 p.

11. Tuan, P. Q. 2006. Spational Adaptivity in Superesolution of Under-Sampled Image Sequences. Ph.D. Thesis, Delft Technik University, 134 p.

12. Cho, T. S.; Levin, A.; Durand, F.; Freeman, W. T. 2010. Motion blur removal with orthogonal parabolic exposures, IEEE International Conf. on Computational Photography (ICCP).

13. Levin, A.; Sand, P.; Cho, T. S.; Durand, F.; Freeman, W. T. 2008. Motion-Invariant Photography, SIGGRAPH, ACM Transactions on Graphics.
14. Favaro, P.; Soatto, S. 2006. 3-d Shape Estimation and Image Restoration: Exploiting Defocus and Motion blur, Springer-Verlag, 263 p.

15. Brucas, D.; Giniotis, V.; Augustinavicius, G.; Stepanoviene, J. 2010. Calibration of the multiangular prism (polygon), Mechanika 4(84): 62-66.

16. Stauton, R. C. 1998. Edge operator error estimation incorporating measurements of CCD TV camera transfer function, IEE Proceedings Visual Image Signal Processing, 145: 229-235.

17. Trimeche, M. 2006. Super-Resolution Image Reconstruction Using Non-Linear Filtering Techniques. Ph.D. Thesis, Tampere University of Technology, $127 \mathrm{p}$.

18. Truchetet, F.; Nicolier, F.; Laligant, O. 2001. Subpixel edge detection for dimensional control by artificial vision, Journal of Electronic Imaging, 10: 234-239.

R. Lazdinas, V. Vekteris, M. Jurevicius, V. Turla,

E. Jurkonis

AN ANALYSIS OF THE OPTICAL SYSTEM OF A LENGTH MEASUREMENT COMPARATOR

$\mathrm{S} \mathrm{u} \mathrm{m} \mathrm{m} \mathrm{a} \mathrm{r} \mathrm{y}$

In the paper, the optical system of a precise bar length measuring comparator is analysed. The requirements for such a system are determined and systematized. The impact of the resolution, signal discretization frequency, image blurring, bar edge measurement indeterminacy and camera noise on measuring the bar width and establishing the bar position is discussed upon. Various algorithms have been analysed and finally a bar edge identification algorithm oriented to the scale calibration accuracy and the high processing speed was proposed. In the end of the paper, conclusions are provided.

Keywords: comparator, bar length measures, linear scales, raster scales, dynamic calibration.

Received October 31, 2019

Accepted December 01, 2020

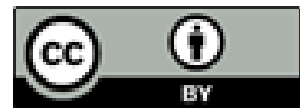

This article is an Open Access article distributed under the terms and conditions of the Creative Commons Attribution 4.0 (CC BY 4.0) License (http://creativecommons.org/licenses/by/4.0/). 